\title{
Treatment of visceral leishmaniasis in south-eastern Nepal: decreasing efficacy of sodium stibogluconate and need for a policy to limit further decline
}

\author{
S. Rijal ${ }^{1}$, F. Chappuis ${ }^{2}$, R. Singh ${ }^{1}$, P. A. Bovier ${ }^{2}$, P. Acharya $^{1}$, B. M. S. Karki ${ }^{1}$, M. L. Das ${ }^{1}$, P. Desjeux ${ }^{3}$, \\ L. Loutan ${ }^{2}$ and S. Koirala ${ }^{1}{ }^{1} B$. P. Koirala Institute of Health Sciences, Dharan, Nepal; ${ }^{2}$ Department of Community \\ Medicine, Geneva University Hospital, 1211 Geneva 14, Switzerland; ${ }^{3}$ Communicable Disease Surveillance and Response \\ (CSR), World Health Organization, 20 Avenue Appia, 1211 Geneva 27, Switzerland
}

\begin{abstract}
Sodium stibogluconate (SSG) is the first-line therapy for visceral leishmaniasis (VL) in south-eastern Nepal. Recent studies from the neighbouring state of Bihar, India, have shown a dramatic fall in cure rates with treatment failure occurring in up to $65 \%$ of VL patients treated with SSG. A prospective study was conducted at a tertiary-level hospital located in south-eastern Nepal from July 1999 to January 2001. Parasitologically proven kala-azar patients with no previous history of treatment for VL were treated with SSG $20 \mathrm{mg} / \mathrm{kg} / \mathrm{d}$ for $30 \mathrm{~d}$ which was extended to $40 \mathrm{~d}$ in those with persistent positive parasitology. Of the 110 patients who completed SSG therapy and were assessed at 1 and 6 months, definite cure was achieved in 99 patients $(90 \%)$ and SSG failure occurred in 11 patients $(10 \%)$. Except for the presence of hepatomegaly and a lower platelet count there was no clinical or laboratory baseline characteristic associated with treatment failure. A significantly lower cure rate $(76 \%, P=0.03)$ was observed in patients from the district of Saptari, which borders the antimony-resistant VL areas of Bihar. The efficacy of SSG as a first-line treatment for VL in south-eastern Nepal was still satisfactory, except for the patients living closer to the antimony-resistant VL areas of India. These findings indicate that the spread of resistance to antimonials is already taking place in Nepal and that a policy to control further spread should be urgently implemented.
\end{abstract}

Keywords: visceral leishmaniasis, Leishmania donovani, chemotherapy, sodium stibogluconate, drug resistance, Nepal

\section{Introduction}

Visceral leishmaniasis (VL) or kala-azar affects an estimated 500000 persons every year worldwide, with $50 \%$ of cases reported from Bangladesh, India, and Nepal (Desjeux, 2001). Visceral leishmaniasis is considered a major health problem in Nepal and is endemic in 12 districts of the eastern and central part of the southern Teraï region, where an estimated 6 million people are at risk of acquiring the disease (Ministry of Health, Nepal, 1999/2000). This region borders the northern part of the state of Bihar in India, which has been the epicentre of the Indian epidemic.

In Nepal, as in most VL-endemic regions, the firstline treatment relies on pentavalent antimony, such as sodium stibogluconate (SSG), as recommended by the WHO (1990). In spite of the inconvenience of prolonged parenteral therapy and potential for severe adverse effects SSG is still used mainly due to its proven efficacy and, when generic compounds are used, affordability (Veeken et al., 2000). However, in the state of Bihar in India, there has been an increasing resistance to SSG since the early 1980 s despite a gradual increase of the dose and duration of treatment up to the maximum recommended dose of $20 \mathrm{mg} / \mathrm{kg} / \mathrm{d}$ for $30 \mathrm{~d}$ (Sundar, 2001). In 1991-92, cure rates of $60 \%$ and $64 \%$ were found in 2 separate studies using this maximum recommended dose (Jha et al,, 1992; Sundar et al., 1995). This trend has shown a further decline and a recent study has reported a long-term cure rate of only $35 \%$ (Sundar et al., 2000a). Thus, SSG has now been abandoned and replaced by the more expensive amphotericin B as the first-line therapy for VL in Bihar.

In Nepal, data on the efficacy of SSG is poorly documented. There has been only 1 published study (Karki et al., 1998) that compared SSG $20 \mathrm{mg} / \mathrm{kg} / \mathrm{d}$ given for $20 \mathrm{~d}$ compared with $30 \mathrm{~d}$ in 2 groups of 27 patients each. The definite cure rate was found to be $78 \%$ and $93 \%$, respectively.

One of the important contributing factors to the drug resistance in Bihar has been attributed to the use of

Address for correspondence: Dr F. Chappuis, Travel and Migration Medicine Unit, Geneva University Hospital, rue Micheli-du-Crest 24, 1211 Geneva 14, Switzerland; phone +41223729620, fax +41223729626 ,

e-mail francois.chappuis@hcuge.ch infra-therapeutic doses and/or insufficient duration of SSG therapy (Sundar, 2001). This phenomenon also exists in Nepal. Moreover, the socio-cultural similarity and the open border between southern Nepal and northern Bihar facilitate cross-border population movements which may also play an important role in the spread of SSG-resistant strains of Leishmania donovani. The objective of the study was to obtain the current status of SSG efficacy in the VL-endemic regions of eastern Nepal and to look for any factors associated with drug resistance. A policy for the control of SSG resistance in Nepal is discussed.

\section{Materials and Methods}

Study site

This study was conducted at the B. P. Koirala Institute of Health Sciences (BPKIHS), a 650-bed University Hospital located $2 \mathrm{~km}$ from the town of Dharan, Sunsari district in the Eastern region of Nepal. The BPKIHS serves as a reference tertiary-level hospital for the Eastern region which includes several VLendemic districts. Recruitment of patients took place at the outpatient department and the emergency room of BPKIHS from July 1999 to August 2000. Patients came directly to BPKIHS or were referred from the district hospitals. The Ethical Committee of BPKIHS approved the research protocol in May 1999.

\section{Inclusion and exclusion criteria}

Parasitologically proven VL cases with no history of previous treatment with SSG were included after obtaining informed consent from the patient or his/her guardian. Only patients from the 3 neighbouring districts of Sunsari, Morang, and Saptari were included as follow-up would not be practically possible for patients coming from more remote districts. There was no other exclusion criteria.

\section{Initial evaluation}

All patients with suspected VL (history of fever $\geqslant 2$ weeks duration and clinical splenomegaly) were admitted to the medical or paediatric ward for a complete examination. This included clinical evaluation, complete blood count, chemistry, blood culture, urine analysis, and chest radiography. HIV testing was done after pre-test counselling using Vironostica ${ }^{\mathbb{R}}$ and Re- 
combigen $^{\mathbb{Q}}$ enzyme-linked immunosorbent assay tests. Diagnosis of VL was made by Giemsa-stained bone marrow aspirate or spleen aspirate (if bone marrow aspirate was negative). Stained smears were designated as 'positive' if $L$. donovani bodies were seen or 'negative' if none were seen in 100 oil immersion fields. Two doctors read the slides independently. Parasite density score was determined microscopically in the Giemsastained smears by the use of a logarithmic scale ranging from 0 (no parasites per 100 oil immersion fields) to +6 ( $>100$ parasites per field). For quality control, $10 \%$ of the slides were cross-checked at the Protozoology Unit (Prof. Dominique Le Ray) of the Institute of Tropical Medicine in Antwerp, Belgium.

\section{Treatment}

All the patients included were allocated into 2 treatment groups: Group A received the full treatment course in the hospital and Group B received the first 5$7 \mathrm{~d}$ treatment in the hospital and the rest as outpatients at the nearest health facility to their place of residence. Allocation into the 2 groups was made according to patients' preference as random allocation was not possible. Patients were treated with generic SSG (sodium antimony gluconate [SAG] from Albert David Ltd, Calcutta, India) $20 \mathrm{mg} / \mathrm{kg} / \mathrm{d}$ for $30 \mathrm{~d}$ as recommended by the Nepalese National Guidelines.

\section{Follow-up}

Clinical assessment and parasitological examination for $L$. donovani bodies was repeated at the end of $30 \mathrm{~d}$ of SSG therapy (1-month follow-up). If $L$. donovani bodies were still present at this time, SSG was extended for another $10 \mathrm{~d}$. If at the end of this extended period $L$. donovani bodies were still found in tissue aspirate, the patient was then treated with the second-line therapy amphotericin $\mathrm{B}, 0.5 \mathrm{mg} / \mathrm{kg} / \mathrm{d}$ for $14 \mathrm{~d}$. All patients were followed-up at 3 and 6 months for further clinical evaluation. Parasitological examination was repeated at 6 months in all patients and at 3 months if relapse was clinically suspected (fever, spleen enlargement). The patients were followed-up at the BPKIHS or actively in their homes if they did not attend the BPKIHS on the fixed date. All relapse cases were treated with amphotericin B $0.5 \mathrm{mg} / \mathrm{kg} / \mathrm{d}$ for $14 \mathrm{~d}$.

\section{Case definitions}

We used the following definitions: VL case, patient with clinical signs (prolonged fever and splenomegaly) and positive for $L$. donovani bodies in tissue aspirate (bone marrow or spleen); initial cure, a VL case with absence of fever and negative parasitology at the end of SSG therapy; non-responder, a VL case with positive parasitology after $40 \mathrm{~d}$ of SSG therapy; definitive cure, a VL case with no clinical signs of relapse and negative parasitology at 6 months follow up; relapse case, a VL case with initial cure but with reappearance of clinical signs and positive parasitology during the 6 months follow-up; and SSG failure, non-responder or relapse case.

\section{Statistical analysis}

Frequencies and cross-tabulations were used to describe the proportions of treatment failure across baseline socio-demographic and medical characteristics. Mean and median values were used for continuous characteristics such as age or duration of fever. To study the effect of place of stay, logistic regression was used to adjust for other significant socio-demographic and medical factors. Pearson's $\chi^{2}$, independent sample $t$ tests, and Mann-Whitney $U$ tests were used to test the statistical differences across groups, where appropriate. All tests were two-tailed, with a significance level of 0.05 .

\section{Results}

Between July 1999 and August 2000, 120 VL patients were included in the study and started on SSG therapy. Four patients $(3.3 \%)$ died during the course of treatment: 2 from cardiotoxicity (electrocardiographicproven arrhythmia), 1 from septic shock and 1 patient with underlying psychiatric illness who committed suicide during his hospital stay. Two other patients developed evidence of cardiotoxicity during SSG treatment and were switched to amphotericin B. The total incidence of cardiotoxicity due to SSG was thus $3.3 \%$.

Of the 114 patients who completed $30 \mathrm{~d}$ SSG therapy, 103 had a negative parasitology. Eleven remained parasitologically positive and required extension of the SSG therapy to $40 \mathrm{~d}$. Of these 11 , only 1 became parasitologically negative and 10 remained positive after $40 \mathrm{~d}$. Thus, at completion of treatment there were 104 initial cures and 10 non-responders. Four patients who had shown initial cure were lost to follow-up at 6 months. Of the 100 patients analysed at 6 months follow-up, 1 had a positive parasitology (relapse) and 99 remained parasitologically negative (definite cure).

The 10 patients with unknown status of resistance to SSG (death during treatment (4), treatment changed to amphotericin B (2), and lost to follow-up (4)) were excluded from further analysis. Of the remaining 110 patients, SSG failure (non-responders or relapse) was seen in $10 \%(11 / 110)$ and definite cure was achieved in $90 \%(99 / 110)$. Table 1 compares baseline sociodemographic characteristics, clinical signs and laboratory values in the 110 patients followed-up at 6 months.

All the patients were negative to HIV testing. A total of $84(76 \%)$ received the full treatment as in-patients (group A) and $26(24 \%)$ received the initial SSG treatment $(5-7 \mathrm{~d})$ as in-patients and the rest as outpatients in the peripheral health facilities (group B).

Treatment failure was only significantly associated with the place of stay, the presence of hepatomegaly, and a lower platelet count (Table 2). Treatment failure occurred in $24 \%$ of patients coming from the district of Saptari compared with only $5 \%$ for the district of Sunsari and $6 \%$ for the district of Morang $(P=0.03)$. Patients coming from Saptari were more frequently men ( $80 \%$ vs. $53 \%, P=0.02)$, more frequently had a positive blood culture at the time of diagnosis $(20 \% \mathrm{vs}$. $5 \%, P=0.01$ ), and had more parasites in bone marrow examination $(57 \%$ with +++ to +++++ vs. $31 \%$ with + to,$++ P=0.03$ ). However, patients coming from Saptari district did not show any other significant difference in disease severity such as duration of fever, spleen size, and haemoglobin count when compared with patients from the other 2 districts (data not shown). In logistic regression analysis, taking into account the effect of gender, positive blood culture and degree of parasite infestation in the bone marrow, treatment failure was still more frequent for patients coming from Saptari $(\mathrm{OR}=6.8,95 \%$ CI $1.3-37.1)$.

\section{Discussion}

In this study, we found that the current efficacy of SSG in the treatment of VL remains satisfactory in the eastern part of the VL-endemic area of Nepal where $90 \%$ of patients who completed their treatment and 6month follow-up showed a definite cure. Except for the presence of hepatomegaly and a lower platelet count, we did not find any clinical or laboratory findings associated with resistance to SSG, as also reported by Sundar et al. (2000a) in India. Co-infection with HIV, an important cause of treatment failure, was not found in this study.

However, a significantly lower cure rate of $76 \%$ was found in patients coming from the district of Saptari, situated in the western extremity of the study area and closer to the northern districts of the Indian state of Bihar (Figure) where resistance to SSG is at its highest 
Table 1. Comparison of baseline socio-demographic, clinical, and laboratory values in 110 visceral leishmaniasis patients treated with sodium stibogluconate in south-eastern Nepal with definite cure or treatment failure at six months follow-up, July 1999-August 2000

\begin{tabular}{|c|c|c|c|c|}
\hline & $\begin{array}{c}\text { Overall } \\
(n=110) \\
\text { Mean (SD) }\end{array}$ & $\begin{array}{c}\text { Definite cure } \\
(n=99) \\
\text { Mean }\end{array}$ & $\begin{array}{l}\text { Treatment } \\
\text { failure } \\
(n=11) \\
\text { Mean }\end{array}$ & $P^{a}$ \\
\hline \multicolumn{5}{|l|}{ Socio-demographic characteristics } \\
\hline Age (years) & $23.8(13.9)$ & 24.1 & 21.5 & 0.55 \\
\hline \multicolumn{5}{|l|}{ Clinical signs } \\
\hline Duration of fever (weeks) & $7.6(7.0)$ & 7.6 & 7.1 & 0.82 \\
\hline Temperature $\left({ }^{\circ} \mathrm{F}\right)$ & $102.3(1.4)$ & 102.3 & 102.5 & 0.70 \\
\hline Spleen size $(\mathrm{cm})$ & $5.7(3.5)$ & 5.6 & 6.5 & 0.42 \\
\hline \multicolumn{5}{|l|}{ Laboratory test results } \\
\hline Haemoglobin $(\mathrm{g} / \mathrm{dL})$ & $8.9(1.8)$ & 8.9 & 8.4 & 0.39 \\
\hline White blood cells $\left(/ \mathrm{mm}^{3}\right)$ & $4030(1310)$ & 3990 & 4360 & 0.39 \\
\hline Platelets $\left(/ \mathrm{mm}^{3}\right)$ & $148900(73700)$ & 152400 & 117300 & 0.02 \\
\hline Creatinine $(\mathrm{mg} / \mathrm{dL})$ & $0.67(0.24)$ & 0.68 & 0.61 & 0.37 \\
\hline DAT end titre ( 1 missing value $)^{\mathrm{b}}$ & $819200(-)$ & 819200 & 3276800 & 0.37 \\
\hline
\end{tabular}

andependent samples $t$ test.

${ }^{\mathrm{b}}$ Median values and Mann-Whitney $U$ test (DAT end titres were not normally distributed).

Table 2. Relationships between treatment failure assessed at 6 months follow-up and baseline socio-demographic and clinical characteristics of 110 visceral leishmaniasis patients treated with sodium stibogluconate in south-eastern Nepal, July 1999-August 2000

\begin{tabular}{|c|c|c|c|}
\hline & $\begin{array}{c}\text { Overall } \\
(n=110) \\
n(\%)\end{array}$ & $\begin{array}{l}\text { Treatment failure } \\
(n=11) \\
n(\%)\end{array}$ & $P^{\mathrm{a}}$ \\
\hline \multicolumn{4}{|l|}{ Socio-demographic characteristics } \\
\hline Gender & & & 0.33 \\
\hline Male & $65(59.1)$ & $5(7.7)$ & \\
\hline Female & $45(40.9)$ & $6(13.3)$ & \\
\hline Place of stay & & & 0.03 \\
\hline Sunsari & $38(34.5)$ & $2(5.3)$ & \\
\hline Saptari & $25(22.7)$ & $6(24.0)$ & \\
\hline Morang & $47(42.7)$ & $3(6.4)$ & \\
\hline Occupation & & & 0.24 \\
\hline Farmer & $26(23.6)$ & $2(7.7)$ & \\
\hline Housewife & $22(20.0)$ & $1(4.5)$ & \\
\hline Student & $50(45.5)$ & $8(16.0)$ & \\
\hline Other & $12(10.9)$ & 0 & \\
\hline \multicolumn{4}{|l|}{ Symptoms and clinical signs } \\
\hline Weakness & $34(30.9)$ & $5(14.7)$ & 0.27 \\
\hline Weight loss & $76(69.1)$ & $7(9.2)$ & 0.68 \\
\hline Cough & $11(10.0)$ & $2(18.2)$ & 0.34 \\
\hline Abdominal pain & $23(20.9)$ & $3(13.0)$ & 0.58 \\
\hline Vomiting & $5(4.5)$ & $1(20.0)$ & 0.45 \\
\hline Skin darkening & $31(28.2)$ & $4(12.9)$ & 0.53 \\
\hline Lymph nodes & $5(4.5)$ & 0 & 0.45 \\
\hline Hepatomegaly & $76(69.1)$ & $11(14.5)$ & 0.02 \\
\hline \multicolumn{4}{|l|}{ Laboratory test results } \\
\hline BM intensity ( 15 missing values) & & & 0.20 \\
\hline+ to ++ & $60(63.2)$ & $5(8.3)$ & \\
\hline+++ to +++++ & $35(36.8)$ & $6(17.1)$ & \\
\hline Positive blood culture & $9(8.2)$ & $1(11.1)$ & 0.91 \\
\hline Positive malaria smear & $2(1.8)$ & 0 & 0.63 \\
\hline Associated tuberculosis & $3(2.7)$ & $1(33.3)$ & 0.17 \\
\hline Type of care & & & 0.65 \\
\hline In-patient (Group A) & $84(76.4)$ & $9(10.7)$ & \\
\hline Outpatient (Group B) & $26(23.6)$ & $2(7.7)$ & \\
\hline
\end{tabular}

(Sundar et al., 2001). This decreased cure rate observed in patients from Saptari is not likely to be due to differences in patients' characteristics or disease severity. Our results suggest that it is most likely that the decreased response to treatment observed in Saptari district is due to resistant strains of $L$. donovani as recently demonstrated in Bihar (Lira et al., 1999). The resurgence of VL in Nepal started in the early 1980s, a decade after the epidemic began in Bihar, which could explain the current observed difference of SSG efficacy between the 2 countries. The increased failure rate in patients from Nepalese districts neighbouring Bihar strongly suggests that the spread of SSG resistance is taking place in Nepal and it may follow a similar course to that in Bihar if no immediate measures are taken.

The intense cross-border population movement be- 


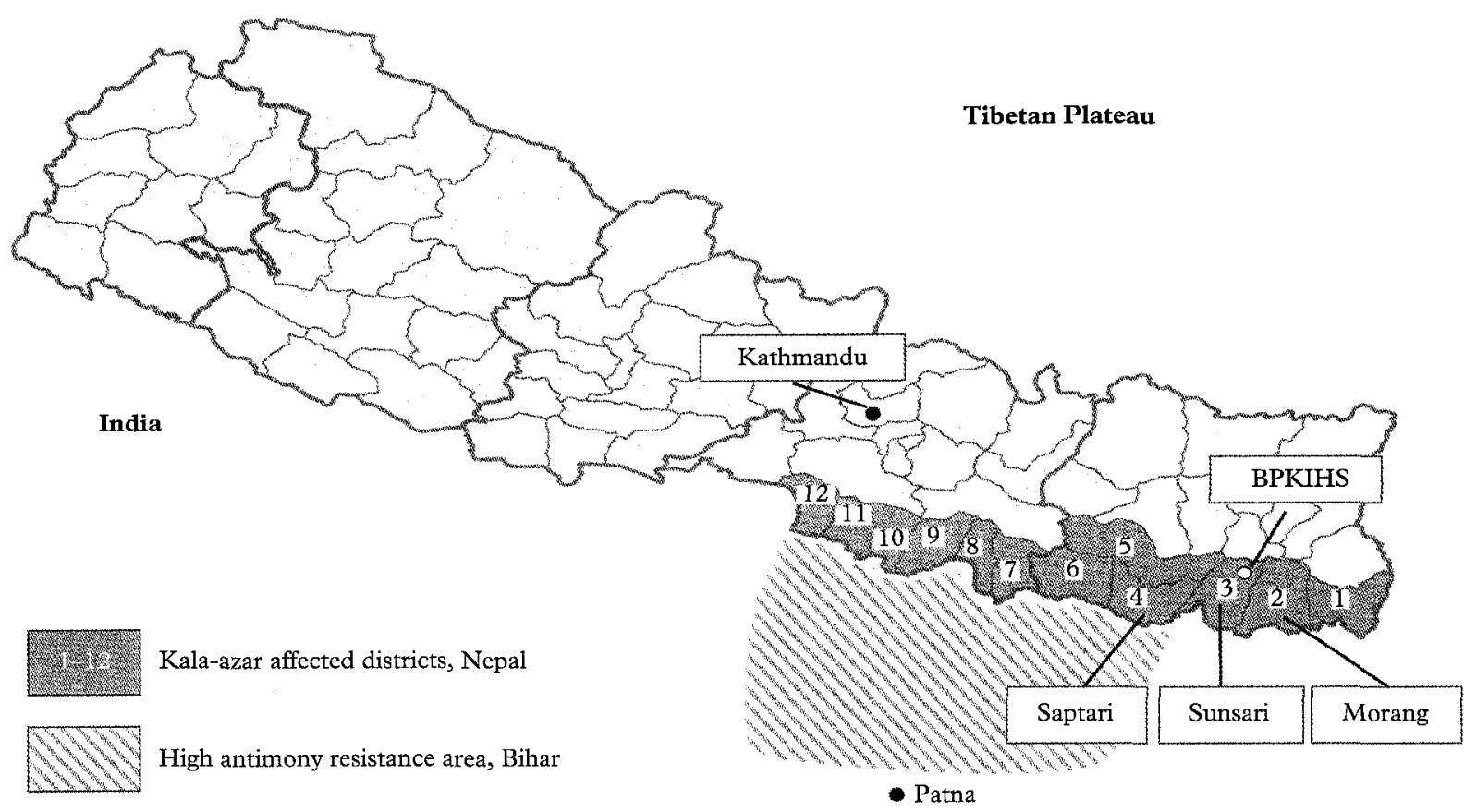

Figure. Map showing the 12 visceral leishmaniasis-endemic districts in south-eastern Nepal and the antimony resistance area in the state of Bihar, India, 1999. BPKIHS, B. P. Koirala Institute of Health Sciences.

tween the state of Bihar and the Terai plain of Nepal could facilitate the spread of the resistant parasites. Moreover, drug misuse, a well-recognized risk factor for the development of resistant parasites (Bryceson et al., 1985), is also common but poorly documented in Nepal: inadequate dosage and/or duration of treatment of SSG is often prescribed in Nepal, mostly by poorly qualified practitioners. In Bihar, Sundar et al. (1994) showed that only $26 \%$ of the 312 patients previously treated with SSG and presenting with a refractory disease had been treated according to the WHO (1996) guidelines.

The current free provision of VL drugs in Nepal to all hospitals located in the endemic region, as part of the National Kala-azar Control Program of the Nepalese Ministry of Health, may be a factor promoting adequate drug use. This may relieve economic pressure on patients to prematurely interrupt treatment but does not solve the constraints of transport and costs of hospitalization. Most VL patients belong to the lowest socio-economic class and have very limited capacity to pay for the cost of treating the disease (Desjeux, 1996). Moreover, the vast majority of VL patients admitted to public hospitals in Nepal receive only the initial few days of SSG until clinical improvement as in-patients and their treatment is completed as outpatients in peripheral health facilities. Considering the long daily walking time to reach health facilities, the painful i.m. injections and the other priorities of daily life, this strategy may lead to inadequate adherence to treatment, subsequently decreasing treatment efficiency and promoting the selection of resistant parasites. In our study, we did not find any significant difference in the outcome of patients between those receiving the full treatment as in-patients and those treated mostly as outpatients. However this study design had limitations, as we were unable to randomize the patients groups.

As suggested by Bryceson (2001), a policy to prevent and control the spread of SSG resistance should be implemented in VL-endemic areas like Nepal. Such a policy should include the reinforcement of current activities aiming to decrease overall disease transmission, measures to prevent drug misuse and implementation, after evaluation, of combination therapies.
The tools available for the control of anthroponotic VL have been recently reviewed by Boelaert et al. (2000). Vector control through insecticide spraying, in association with early case detection and treatment, is an effective way to reduce transmission of the disease (Saxena et al., 1996), and thus also transmission of drug-resistant strains. This requires strong political commitment and is costly. The use of impregnated bednets has the potential to protect healthy people against VL (Bern et al., 2000), and to impede untreated patients to disseminate the parasite through sandfly bites.

Measures to prevent SSG misuse in Nepal could include the widespread implementation of test-treatment algorithms, directly observed therapy for outpatients' care, education of practitioners from both public and private sectors to prescribe adequate treatment schedules, adequate supply of reliable generic SSG with proven efficacy and safety profile to health facilities in the endemic areas, and the patients' access to free or very low-cost drugs.

The use of combination therapy for VL could be an appealing approach for treating patients in drugresistant areas, protecting each component of the combination against selection of resistant mutants and using drugs at lower and thus safer total doses. In the case of malaria, a control of mefloquine resistance has been obtained within a few years of systematic use of the artesunate-mefloquine combination in western Thailand (Nosten et al., 2000). It is currently unclear if a similar strategy for VL would be successful but such an approach should be considered in areas, such as Nepal, where resistance to SSG is increasing. Combinations of SSG and paromomycin have shown to be efficient and safe in India (Thakur et al., 2000), Sudan (Seaman et al., 1993), and Kenya (Chunge et al., 1990) but paramomycin has yet to be registered for VL treatment and its future production and cost remain unclear. Other possible combinations could include in future trials conventional or liposomal amphotericin B as well as miltefosine, an oral drug showed to be very efficient in India when used alone for 4 weeks (Jha et al., 1999; Sundar et al., 2000b). Miltefosine was registered for the treatment of VL in India in 2002 and a 
phase IV trial will take place in India and Nepal in the coming year. However, because of the long half-life of miltefosine and the long duration of treatment, one might fear that $L$. donovani will rapidly develop resistance to this drug if it is used alone.

Regular monitoring of SSG susceptibility in Nepal should be an important component of this policy. It should be performed preferably in vitro using the amastigote-macrophage model, which evaluate the resistance of $L$. donovani isolates to antimonials (Croft, 2001)

The HIV seroprevalence in the general population in Nepal is currently around $0.3 \%$ but risk factors for an increasing prevalence are present (Furber et al., 2002). Monitoring and fighting the progression of HIV disease is very important in this region considering the much higher risk of treatment failure in HIV-VL co-infected patients treated with SSG or with any other anti-VI drugs (Lopez-Velez et al., 1998).

In conclusion, we showed that the efficacy of SSG to treat VL in eastern Nepal remains satisfactory overall but that areas of lower response to SSG exist in this region. Considering the lower price of SSG (in its generic form) and the higher cost and/or lack of availability of present alternative therapies, it is necessary that current efforts in Nepal focus on limiting the spread of resistance to SSG.

\section{Acknowledgements}

This study was funded by grants allocated by the Novartis Foundation (UK), WHO, and Geneva University Hospital (Switzerland)

\section{References}

Bern, C., Joshi, A. B., Jha, S. N., Das, M. L., Hightower, A., Thakur, G. D. \& Bista, M. B. (2000). Factors associated with visceral leishmaniasis in Nepal: bed-net use is strongly protective. American fournal of Tropical Medicine and Hygiene, 63, 184-188.

Boelaert, M., Criel, B., Leeuwenburg, J., Van Damme, W., Le Ray, D. \& Van der Stuyft, P. (2000). Visceral leishmaniasis control: a public health perspective. Transactions of the Royal Society of Tropical Medicine and Hygiene, 94, 465-471.

Bryceson, A. D. M., Chulay, J. D., Ho, M., Mugambii, M., Were, J. B., Muigai, R., Chunge, C., Gachihi, G., Meme, J., Anabwani, G. \& Bhatt, S. M. (1985). Visceral leishmaniasis unresponsive to antimonial drugs. I. Clinical and immunological studies. Transactions of the Royal Society of Tropical Medicine and Hygiene, 79, 700-704.

Bryceson, A. (2001). A policy for leishmaniasis with respect to the prevention and control of drug resistance. Tropical Medicine and International Health, 6, 928-934.

Chunge, C. N., Owate, J., Pamba, H. O. \& Donno, L. (1990). Treatment of visceral leishmaniasis in Kenya by aminosidine alone or combined with sodium stibogluconate. Transactions of the Royal Society of Tropical Medicine and Hygiene, 84, $221-225$.

Croft, S. L. (2001). Monitoring drug resistance in leishmaniasis. Tropical Medicine and International Health, 6, 899-905.

Desjeux, P. (1996). Leishmaniasis. Public health aspects and control. Clinical Dermatology, 14, 417--423.

Desjeux, P. (2001). The increase in risk factors for leishmaniasis worldwide. Transactions of the Royal Society of Tropical Medicine and Hygiene, 95, 239-243.

Furber, A. S., Newell, J. N. \& Lubben, M. M. (2002). A systematic review of current knowledge of HIV epidemiology and of sexual behaviour in Nepal. Tropical Medicine and International Health, 7, 140-148.

Jha, T. K., Singh, N. K. P. \& Jha, S. (1992). Therapeutic use of sodium stibogluconate in kala-azar from some hyperendemic districts of N. Bihar, India [Abstract]. Fournal of the Association of Physicians of India, 40,868 .

Jha, T. K., Sundar, S., Thakur, C. P., Bachmann, P., Karbwang, J., Fischer, C., Voss, A. \& Berman, J. (1999). Miltefosine, an oral agent, for the treatment of Indian visceral leishmaniasis. New England foumal of Medicine, 341, $1795-1800$

Karki, P., Koirala, S., Parija, S. C., Handsak, S. G. \& Das, M. L. (1998). A thirty day course of sodium stibogluconate for treatment of kala-azar in Nepal. Southeast Asian foumal of Tropical Medicine and Public Health, 29, 154-158.

Lira, R., Sundar, S., Makharia, A., Kenney, R., Gam, A., Saraiva, E. \& Sacks, D. (1999). Evidence that the high incidence of treatment failure in kala-azar is due to the emergence of antimony resistant strains of Leishmania donovani. Foumal of Infectious Diseases, 180, 564-567.

Lopez-Velez, R., Perez-Molina, J. A., Guerrero, A., Baquero, F., Villarrubia, J., Escribano, L., Bellas, C., Perez-Corral, F. \& Alvar, J. (1998). Clinicoepidemiologic characteristics, prognostic factors, and survival analysis of patients coinfected with human immunodeficiency virus and Leishmania in an area of Madrid, Spain. American foumal of Tropical Medicine and Hygiene, 58, 436-443.

Ministry of Health, Nepal (1999/2000). Annual Report. Kalaazar Control. Kathmandu: His Majesty's Government of Nepal, Ministry of Health, Directorate of Health Services, Epidemiology and Disease Control, pp. 119-124.

Nosten, F., Van Vugt, M., Price, R., Luxenburger, C., Thway, K. L., Brockman, A., McGready, R., Ter Kuile, F., Looareesuwan, S. \& White, N. (2000). Effects of artesunatemefloquine combination on incidence of Plasmodium falciparum malaria and mefloquine resistance in western Thailand: a prospective study. Lancet, 356, 297-302.

Saxena, N. B., Aggarwal, V., Dhillon, G. P., Sharma, R. S. \& Rao, J. S. (1996). Visceral leishmaniasis control in India through primary health care system-a successful experiment of district level planning. Fournal of Communicable Disease, 28, 122-128.

Seaman, J., Pryce, D., Sondorp, H. E., Moody, A., Bryceson, A. D. M. \& Davidson, R. (1993). Epidemic visceral leishmaniasis in Sudan: a randomized trial of aminosidine plus sodium stibogluconate versus sodium stibogluconate alone. Foumal of Infectious Diseases, 168, 715-720.

Sundar, S. (2001). Drug resistance in Indian visceral leishmaniasis. Tropical Medicine and International Health, 6, $849-854$.

Sundar, S., Thakur, B. B., Tandon, A. K., Agrawal, N. R., Mishra, C. P., Mahapatra, T. M. \& Singh, V. P. (1994). Clinico-epidemiological study of drug resistance in Indian kala-azar. British Medical fournal, 308, 307.

Sundar, S., Rosenkaimer, F., Lesser, M. L. \& Murray, H. W. (1995). Immunochemotherapy for a systematic intracellular infection: accelerated response using interferon $-\gamma$ in visceral leishmaniasis. Fournal of Infectious Diseases, 171, 992-996.

Sundar, S., More, D. K., Singh, M. K., Singh, V. P., Sharma, S., Makharia, A., Kumar, P. C. K. \& Murray, H. (2000a). Failure of pentavalent antimony in visceral leishmaniasis in India: report from the center of the Indian epidemic. Clinical Infectious Diseases, 31, 1104-1107.

Sundar, S., Makharia, A., More, D. K., Agrawal, G., Voss, A., Fischer, C., Bachmann, P. \& Murray, H. (2000b). Short course oral miltefosine treatment for visceral leishmaniasis. Clinical Infectious Diseases, 31, 1110-1112.

Thakur, C. P., Kanyok, T. P., Pandey, A. K., Sinha, G. P., Zaniewski, A. E., Houlihan, H. H. \& Olliaro, P. (2000). A prospective randomized, comparative, open-label trial of the safety and efficacy of paromomycin (aminosidine) plus sodium stibogluconate versus sodium stibogluconate alone for the treatment of visceral leishmaniasis. Transactions of the Royal Society of Tropical Medicine and Hygiene, 94, 429-431.

Veeken, H., Ritmeijer, K., Seaman, J. \& Davidson, R. A. (2000). Randomized comparison of branded sodium stibogluconate and generic sodium stibogluconate for the treatment of visceral leishmaniasis under field conditions in Sudan. Tropical Medicine and International Health, 5, $312-317$.

WHO (1990). Control of the Leishmaniases. Report of a WHO expert committee. Geneva: World Health Organization, Technical Report Series, No. 793.

WHO (1996). Manual on Visceral Leishmaniasis Control. Geneva: World Health Orgnization, WHO/LEISH/96.40.

Received 23 fuly 2002; revised 26 November 2002; accepted for publication 28 November 2002 